\title{
Interactive comment on "Estimates of tree root water uptake from soil moisture profile dynamics" by Conrad Jackisch et al.
}

Conrad Jackisch et al.

c.jackisch@tu-braunschweig.de

Received and published: 14 May 2020

\section{General reply to all referees}

We sincerely thank Jesse Nippert, Jia Hu and Leander Anderegg for their intense study of our manuscript and their constructive feedback. We clearly understand that we have to simplify some of the dense writing and figures to convey our findings more clearly. The referees made several detailed suggestions for this, along which we will organise the revisions. We will self-critically check for simplifications of jargon and clarity in our arguments.

With respect to the observed process dynamics of measured and inferred variables, 
we will carefully revise the manuscript towards i) a more detailed description of the observed results, ii) more coherent argumentation lines, and iii) the limits of the presented approach. We will put specific attention to a) the conversion of sap flow velocity and rhizosphere water withdrawal to flux rates, b) the assessment of the coherence of the diurnal signal with the assumed step shape, and c) the reference to inferred matric potential. At a meta-level, we have to make sure not to overstretch the data set at hand which is basically a first reference. We hope that many more researchers will employ, test and evaluate the proposed approach to estimate RWU, which together will form a more comprehensive picture of the complementary information in RWU, SF and ET.

\section{Specific replies to the comment by Jesse Nippert}

The referees' comments are given in italics with our answers in regular font style.

Here the authors use detailed TDR measurements at consecutive depths in the soil profile to infer changes in water uptake by several beech trees on varying substrates. The authors compile a rigorously quantitative set of complimentary data (including sap flux from target trees) to validate the fluxes derived from the soil moisture measurements. Generally, the 'root water uptake' metric derived from soil moisture was similar to sap flux estimates, particularly during periods with greater water availability and on the sandy site. The goal of this study - develop a new metric to provide detailed water-use / transpiration estimates in complex canopies - is novel, rigorous, and has tremendous potential for more detailed ecological investigations.

Thank you very much for this summary in which we see our study well-understood. 
I have few major concerns or suggestion revisions for the authors to address. My most noteworthy suggestion for the authors is to simply / clarify this manuscript whenever possible. This is an extraordinarily detailed and jargon-rich manuscript that needs to be simplified. I'm a scientist that studies plant-water use (so in theory, this manuscript is directly within my field of study) and yet I found myself reading and re-reading sections to try to understand what was done, what the data means, and how the authors derived their conclusions. For many of the figures, I was never able to gain a full appreciation of what was conveyed (what exactly am I seeing in Figures 11 and 12?) and the legends were often non-descriptive (see Fig. 4 for an example). Thus, I encourage the authors to reduce the jargon, better explain the development of the RWU calculation, and simplify the figures whenever possible. The novelty and creativity of this manuscript is in the top 95\%, but the clarity and delivery of the information is in the 50-60th percentile. I'm fully confident the authors can address this issue.

As stated, we fully take this point. Our revisions will take special care to strongly simplify/clarify the language and figures.

Other comments: The Introduction is generally sound, but there are a few items of concern. On page 1 line 17, the authors need to remove 'even over grasslands'. Grasslands are not definitely simpler ecosystems that forests, so please do not refer to them as such.

We agree to the point that grasslands are not simpler ecosystems than forests and that this wording was not well chosen. We will remove it.

On line 18, please address the 'optimise their water transport to respiration'. The optimality theory has been challenged many times since 2009, and in fact doesn't appear to be valid. In addition, why would water transport be optimized for respiration? I believe you meant assimilation. Regardless, please update.

Printer-friendly version

Discussion paper 
You rightly assume that we refer to assimilation. This will be updated. We also take your point that the optimality theory is under debate and that our study does not have the means to become a take on this level. We will reconsider how to make our point that a better knowledge about RWU can support the discussion.

Examples of RWU assessments in the literature are provided on page 2. While I have never read an approach as detailed as the one presented here, transpiration dynamics have been assessed by comparing sap flux with changes in soil moisture dynamics previously. Please check out Holdo and Nippert 2015 Ecology (https://doi.org/10.1890/141986.1) for a study comparing transpiration dynamics using sap flux, soil moisture, isotopes, and changes in canopy temperature among coexisting trees and grasses.

Thank you for pointing to this study.

On page 5/line 7, the authors note that sap flux was monitored in 4 trees near the TDR probes at each site. Do subsequent sap flux data (Fig. 3, 5, 6, etc) represent a single tree, or an interpolation across all 4 trees per site?

Yes, the respective data represents a single tree. In the paragraph P5L8ff. we seek to clarify this. We will add it more clearly that we are only using one tree per site in this paragraph.

Page 5, line 22. 'Efficiency' is misspelled.

Thank you. We will correct this.

Section 3 describes Fig. 3, and the derivation of RWU based on soil moisture step change. How was this 3 day period selected? Can I assume it was the best 3-day period where a step change was observed during this summer? Does the lower corre- 
lation in RWU time series (and during drier times) reflect derivation of the metric during an ideal period, which then loses predictive power during mean summer soil moisture periods?

This exemplary period in Fig. 3 has been chosen arbitrarily as it combines clear sky conditions (day 1), clear sky with an intermediate shading (day 2) and a fair weather with radiation noise by smaller cumulus clouds (day 3). The step changes have been observed at many more days which we account for by calculating the NSE for each day. This is evaluated in Fig. 12. We do not compare other periods with any ideal one but we calculate i) a NSE between the data and an idealised step shape of one day and ii) a KGE between estimates of RWU and SF (Fig. 8). We will seek to clarify the respective references in the revisions.

With respect to our proposed approach, yes, it loses predictive power when the changes in soil moisture become relatively small and hence our assumption of the diurnal steps is no longer met. This changes with time and depth layer. Roughly summarised: We find steps in the data with a NSE persistently $>0$ between May and August and values near 1 between mid May and early July at both sites within the responsive layers. This temporal dynamics was intended to be included in Fig. 6 as shading but for sake of simplification we will remove it from this figure and explain it in greater detail elsewhere.

When linking RWU from TDR data to transpiration dynamics from an individual tree, how did you account for water use by the understory vegetative community? Unless there were no grasses, forbs, saplings, etc. - wouldn't these species be using water from the same soil depths which would then complicate predictions of individual tree water-use using changes in soil moisture? The methods section does not describe the understory.

Both sites did not have any understory vegetation. We will add a sentence on this. With

Printer-friendly version

Discussion paper
Interactive comment

\section{5}


respect to evaporation we expect an effect on the top $20 \mathrm{~cm}$. However, since the signal from this layer is only rarely evaluated as RWU we are quite confident to be correct here. From a discussion point for the proposed approach, we agree that in many applications with understory vegetation the soil moisture dynamics cannot differentiate between the different plants.

On the sandy site, how did you account for capillary action within the soil profile and subsequent evaporation? Or do you have some information (which I may have missed) on evaporation rates, and the depths of the evaporative fronts from these soils?

We do not have any reliable evaporation reference. As stated, we have to expect some evaporation from the top layer. One should however note that we have a litter layer of about $5-8 \mathrm{~cm}$ at the sites (we will add this to the site description). The surface is shaded once the leaves are out.

Fig. 6 is an impressive behemoth. One item of interest to me is that the rainfall history does not appear to have any temporal synchrony with RWU or sap flux. Is this true? And if so, why not?

The strongest correlation between rainfall and SF/RWU comes from the reduced irradiation during precipitation periods. At the slate site, we have a late activity end of September after a rainy period. Similarly, we can discern periods of stronger SF/RWU after rain spells over the summer. If this is a generally observable relationship remains unclear within the scope of this study. However, it would be rather plausible that the tree can source water when it is more easily available and the radiative forcing drives the "photosynthesis engine".

In Fig. 7, how does these predictions of water use in $\mathrm{mm} /$ day compare to estimates of Beech from similar locations within the literature. It would be nice to know if these 
predictions fall in the range reported elsewhere.

We will try to give some literature values for comparison.

Page 14, line 9 - I don't think 'ambivalent' is the correct term. An ambivalent picture suggests this was inconclusive research. You concluded many things, and illustrate a path forward for using soil moisture to infer plant water use.

Thank you for pointing to this improper wording. We will revise it.

Page 17, line 5. How do you know it's a minor effect? HR can be quite substantial in many ecosystems.

We agree that hydraulic redistribution in the rhizosphere can be substantial and that especially at the sandy site we might miss important factors by neglecting it. We will rephrase this.

Page 17, line 14 - How would cosmic ray measurements be appropriate here? I was under the impression that cosmic ray data pertains to the top $10 \mathrm{~cm}$ of the soil only.

We refer to attempts of combining cosmic ray measurements with in-situ soil moisture measurements to overcome the point information towards a better spatial representation like the authors in Nguyen et al. (2019) propose. We will rephrase the sentence and stress the combination of methods to clarify.

In the Discussion section, the authors note that RWU and sap flux were not interchangeable, but were complementary. The language infers that RWU may be more desirable than sap flux for estimating transpiration under certain conditions. This seems a bit misleading to me. Sap flux directly measures a physiological process that relates 
to canopy transpiration, while RWU is an inferred metric. Under what conditions would the data from RWU be preferred over sap flux? Under what conditions does RWU outperform sap flux for predictions of transpiration?

We do not think that RWU is more informative than sap flow. Certainly both have their merits and drawbacks. We aim to suggest to refer to both means as complementary gauges of a highly interlinked process. Unfortunately, SF does not directly measure the physiological process but a flow velocity over a more or less difficult to guess crosssection. Similarly, RWU is likely rather heterogeneous when considering the moisture changes in the rhizosphere as a 3D space. Hence an estimate based on one profile has clear limitations, too. We can follow the argumentation that the tree trunk is at least some sort of gauge where all water must pass. However, the SF processing involves quite some steps where the resulting fluxes can scale considerably.

\section{Bibliography}

Nguyen, H. H., Jeong, J., and Choi, M.: Extension of cosmic-ray neutron probe measurement depth for improving field scale root-zone soilmoisture estimation by coupling with representative in-situ sensors, Journal of Hydrology, 571, 679-696, https://linkinghub.elsevier.com/10retrieve/pii/S0022169419301751, 2019.

Interactive comment on Biogeosciences Discuss., https://doi.org/10.5194/bg-2019-466, 2019. 